Oliveira, BB, Heineck, LFM, Cirino, MAG, Morais, JMP, Silva, EM, Barboza, EN \& Pereira, SLO (2020). Didactic games for rockline teaching in Civil Engineering. Research, Society and Development, 9(7): 1-19, e525974425.

\title{
Jogos didáticos para ensino de linha de balanço na Engenharia Civil
}

Didactic games for rockline teaching in Civil Engineering

Juegos didácticos para la enseñanza de líneas de rock en Ingeniería Civil

Recebido: 11/05/2020 | Revisado: 12/05/2020 | Aceito: 15/05/2020 | Publicado: 25/05/2020

\section{Bruno Barbosa de Oliveira}

ORCID: https://orcid.org/0000-0002-1279-1431

Universidade Regional do Cariri, Brasil

E-mail: bruno.barbosa@urca.br

Luiz Fernando MähImann Heineck

ORCID: https://orcid.org/0000-0001-7275-4184

Centro de Estudos Sociais Aplicados da Universidade Estadual do Ceará, Brasil

E-mail: heineck@pq.cnpq.br

Miguel Adriano Gonçalves Cirino

ORCID: https://orcid.org/0000-0001-5441-8080

Universidade Regional do Cariri, Brasil

E-mail: miguel.goncalves@urca.br

João Marcos Pereira de Morais

ORCID: https://orcid.org/0000-0001-8097-9607

Universidade Regional do Cariri, Brasil

E-mail: joaomarcostecnologo@gmail.com

Eduarda Morais da Silva

ORCID: https://orcid.org/0000-0001-9463-8280

Instituto Federal de Educação, Ciência e Tecnologia do Ceará, Brasil

E-mail: eduardamoraisengenharia@gmail.com

Eliezio Nascimento Barboza

ORCID: https://orcid.org/0000-0001-8100-9389

Instituto Federal de Educação, Ciência e Tecnologia do Ceará, Brasil

E-mail: eliezio1999@outlook.com

Synardo Leonardo de Oliveira Pereira

ORCID: https://orcid.org/0000-0003-0789-3094 
Universidade Regional do Cariri, Brasil

E-mail: Synardo.pereira@urca.br

\title{
Resumo
}

A utilização de jogos e simulações é um método alternativo de ensino que pode tornar o aprendizado mais eficaz por meio da relação entre conhecimento e prática, sem os riscos de falha das situações reais. Os jogos e simulações também podem ser utilizados como instrumentos de auxílio em treinamentos, de modo a formar as competências necessárias onde o conhecimento atual não é suficiente. Nesse sentido, este trabalho tem como objetivo discutir o uso do jogo de Lego System ${ }^{\circledR}$ para o ensino da técnica de linha de balanço para alunos de engenharia, utilizado na disciplina de planejamento e custos do curso de pós-graduação em engenharia civil da Universidade Federal do Ceará. Para isso foi realizada uma simulação de planejamento de construção de casas com jogos: constou da construção de um conjunto de quatro casas. Utilizou-se conceitos como programação de serviços com intensa fase de planejamento, sistema de pallets para materiais, durações das atividades, organização do chão de fábrica (layout do canteiro), padronização de serviços, uso kambans de transporte e produção. Constatou-se que a utilização de jogos para o ensino da programação de obra com de linha de balanço facilita a compreensão dos conceitos teóricos e sua aplicação prática.

Palavras-chave: Jogos, Linha de balanço e Ensino de Engenharia.

\begin{abstract}
The use of games and simulations is an alternative method of teaching that can make learning more effective through the relationship between knowledge and practice, without the risks of failure of real situations. Games and simulations can also be used as training aid instruments in order to form the necessary skills where current knowledge is not sufficient. In this sense, this work aims to discuss the use of the Lego System game ${ }^{\circledR}$ for the teaching of the balance line technique for engineering students, used in the discipline of planning and costs of the postgraduate course in civil engineering at the Federal University of Ceará. For this purpose, a simulation of planning the construction of houses with games was carried out: it consisted of the construction of a set of four houses. Concepts such as service scheduling with intense planning phase, pallet system for materials, durations of activities, organization of the shop floor (layout of the construction site), standardization of services, use of transport kambans and production were used. It was found that the use of games for teaching work programming with balance line facilitates the understanding of theoretical concepts and their practical application.
\end{abstract}


Keywords: Games, Balance line; Engineering teaching.

\section{Resumen}

El uso de juegos y simulaciones es un método alternativo de enseñanza que puede hacer que el aprendizaje sea más eficaz a través de la relación entre el conocimiento y la práctica, sin los riesgos de fracaso de situaciones reales. Los juegos y simulaciones también se pueden utilizar como instrumentos de ayuda de entrenamiento para formar las habilidades necesarias cuando los conocimientos actuales no sean suficientes. En este sentido, este trabajo tiene como objetivo discutir el uso del juego Lego System ${ }^{\circledR}$ para la enseñanza de la técnica de línea de equilibrio para estudiantes de ingeniería, utilizado en la disciplina de planificación y costos del curso de postgrado en ingeniería civil en la Universidad Federal de Ceará. Para ello, se llevó a cabo una simulación de planificación de la construcción de casas con juegos: consistía en la construcción de un conjunto de cuatro viviendas. Se utilizaron conceptos como la programación de servicios con fase de planificación intensa, el sistema de palet para materiales, la duración de las actividades, la organización de la planta (disposición del sitio de construcción), la estandarización de los servicios, el uso de kambans de transporte y la producción. Se encontró que el uso de juegos para la enseñanza de la programación de trabajo con línea de equilibrio facilita la comprensión de los conceptos teóricos y su aplicación práctica.

Palabras clave: Juegos, Línea de equilibrio y Enseñanza de Ingeniería.

\section{Introdução}

A utilização de jogos e simulações é um método alternativo de ensino que pode tornar o aprendizado mais eficaz por meio da relação entre conhecimento e prática, sem os riscos de falha das situações reais (Depexe et al., 2006). Os jogos e simulações também podem ser utilizados como instrumentos de auxílio em treinamentos, de modo a formar as competências necessárias onde o conhecimento atual não é suficiente (Rauch-Geelhaar, Jenke \& Thurnes, 2003).

Entretanto, como ressaltam Doyle e Brown (2000), os jogos não devem ser utilizados como única forma de ensino, mas sim como complemento e reforço das lições. O uso de jogos permite que os estudantes utilizem seus conhecimentos e teorias já aprendidas em situações que simulam a realidade, de modo a proporcionar um equilíbrio entre teoria e prática (Depexe et al., 2006). 
Com a aplicação de jogos didáticos a visualização dos processos passa a ser facilitada, essa técnica não auxilia somente professores e alunos, abrangendo também setores executivos a desenvolver novas estratégias, pois a facilidade de manipulação possibilita grandes variações em curtos espaços de tempo sem a necessidade de grandes investimentos e também sem afetar o desempenho fabril (Depexe et al., 2006).

A natureza repetitiva da construção de conjuntos habitacionais, em conjunto com a ênfase da indústria da construção na padronização de processos e modularização de componentes ao longo dos últimos anos tem impulsionado o desenvolvimento de várias técnicas e estratégias de planejamento para estes tipos de construções, tanto a nível macro (tático) como a nível micro (operacional). O conceito fundamental de todas estas técnicas é a Linha de Balanço (Line of Balance - LOB) (Lumsden, 1968; Lutz, 1990).

A técnica de Linha de Balanço foi desenvolvida para atender as necessidades de planejamento da indústria de manufatura e posteriormente foi adaptada e utilizada com sucesso por empresas de construção na Europa (Dressler, 1974), Canadá (Sumichrast \& Russell, 1990) entre outros países. Os maiores benefícios da técnica de Linha de Balanço são: proporciona informações de produção e duração para cada processo repetitivo num formato gráfico que é facilmente interpretado tanto pelo pessoal de planejamento como de obra; facilita a programação da continuidade de trabalho das equipes ao longo das repetições nas unidades (Mendes JR, 2000).

A literatura apresenta diversos casos de aplicação de jogos e simulações como instrumentos de ensino de Linha de Balanço. Por exemplo, Vargas, Krüger, Heineck e Coelho (1998) descrevem uma aplicação de jogos para a simulação da construção de obras prediais. A simulação consta da execução de todas as etapas necessárias para a construção de banheiros sociais de um edifício de dez pavimentos em modelo reduzido confeccionado em cartolina. A execução foi planejada com auxílio da técnica da Linha de Balanço. O objetivo da simulação é aplicar as técnicas de avaliação de produtividade e medição de perdas, além de evidenciar a aplicação de técnicas modernas de gerenciamento de obras.

Vargas, Mendes Júnior, Heineck e Krüger (1998) apresentam melhorias no modelo anterior, com a simulação da construção de um conjunto de casas em escala reduzida, confeccionadas em cartolina colorida. A simulação procura demonstrar as vantagens da utilização da técnica da Linha de Balanço. Para isso, os autores apresentam dois exercícios. No primeiro, os participantes executam todas as etapas do edifício, sem qualquer planejamento. No segundo exercício, utiliza-se o dimensionamento das equipes segundo a técnica da Linha de 
Balanço, o que acarreta menor variabilidade e aumento da produtividade, devido à redução dos tempos improdutivos e auxiliares.

Santos, Borges, Prado e Heineck (2002) apresentam um jogo que simula a construção de um edifício de dez pavimentos que permite avaliar fatores como velocidade, qualidade e desperdícios. Além de abordar a técnica de programação da Linha de Balanço, o jogo permite a avaliação de elementos como a compatibilização de projetos, construtibilidade, estratégias de mercado, trabalho em equipe e estratégia de produção.

Depexe et. al. (2006) apresentam uma simulação de planejamento e construção de um conjunto de 16 casas com jogos de montar. Elaboram o planejamento utilizando a técnica da Linha de Balanço. A montagem das casas é realizada com controle do tempo de execução de cada etapa, para confronto em tempo real com a programação. A realização da simulação possibilitou uma melhor compreensão e utilização prática dos conceitos, em uma situação que simula a realidade de forma simplificada.

Portanto, os jogos e simulações são utilizados para o ensino de diversas áreas do conhecimento, tanto sob a forma de jogos com peças físicas quanto em jogos computacionais. Desta forma, o presente trabalho apresenta o relato da utilização de peças de jogo de lego system ${ }^{\circledR}$ para simulação de programação de obra com linha de balanço e construção um conjunto de quatro casas. Os conceitos da técnica de uso da linha de balanço foram facilitados em sua aprendizagem e assimilação com o uso de jogos.

\section{Metodologia}

A simulação foi realizada na sala de estudos da pós-graduação, fixando o canteiro de obras numa mesa central para atender à característica dos canteiros de obra da construção civil que é o arranjo posicional - obra fixa com material, equipamentos e mão-de-obra ao redor. Foram colocadas três filmadoras para registro posterior dos tempos reais de execução de cada serviço bem como do transporte de material. A equipe de execução da obra foi constituída por cinco (05) alunos da pós-graduação, divididos entre equipe de serviço para cada atividade e setor de suprimento responsável pela entrega dos pallets de material ao receber o kamban de produção.

A equipe de alunos utilizou-se das atividades de programação real da obra, como duração das atividades para definição de linha de balanço com o tempo estimado, ferramentas de suprimento com uso de pallets para material organizado por atividade, uso de kambans de transporte e produção, organização do chão de fábrica (layout do canteiro) para execução das 
casas de acordo com uma numeração e ordem pré-definidas, produção puxada das atividades, projeto das casas.

\subsection{Simulação}

Na simulação foi programada a construção de quatro casas.

\subsubsection{Projeto executivo}

Foi elaborado um único projeto executivo, baseado na modulação das peças (Figura 1) para quatro casas, sendo o mesmo esmiuçado pela equipe durante a fase de planejamento como meio de encontrar uma forma que execução que mais se aproximasse de uma canteiro-de-obras.

Figura 1: Projeto executivo das quatro casas.

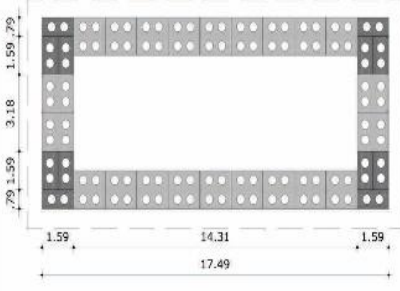

01 - FUNDAÇÃO

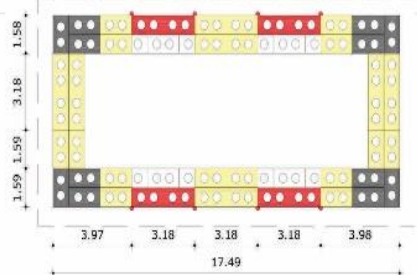

02 - 1a FIADA - ALVENARIA

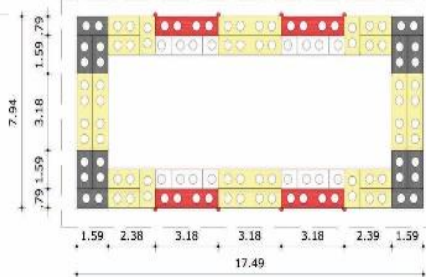

03 - 2a. FIADA - ALVENARIA

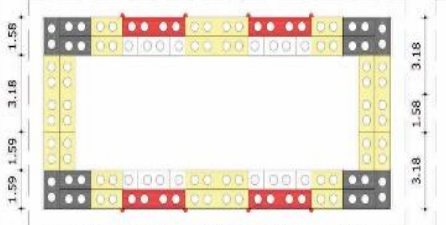

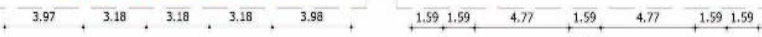

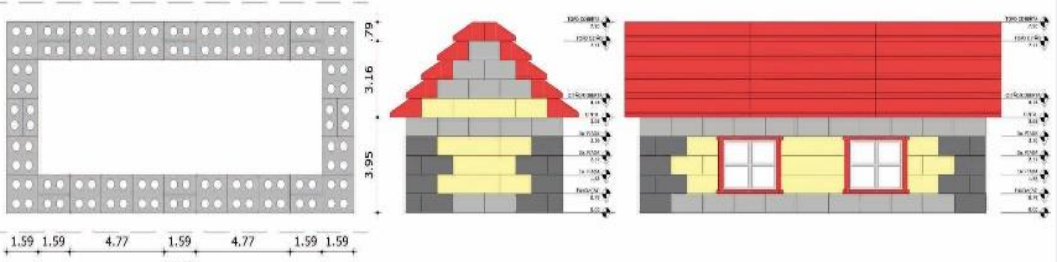

04 - 3a FIADA - ALVENARIA 05 - CINTA DE AMARRAÇÃO

06- FACHADAS LATERAL E FRONTAL

Fonte: Autores, 2019.

\subsubsection{Programação da obra}

Após decisão consensual durante a fase de planejamento, adotou-se a seguinte sequência: fundação; alvenaria de vedação $1^{\mathrm{a}}$ fiada e esquadria; alvenaria de vedação $2^{\mathrm{a}}$ fiada; alvenaria de vedação $3^{\mathrm{a}}$ fiada; cinta de amarração e coberta. Na Figura 2 a sequência dos serviços a serem executados para cada casa. 
Figura 2: Sequência de montagem.

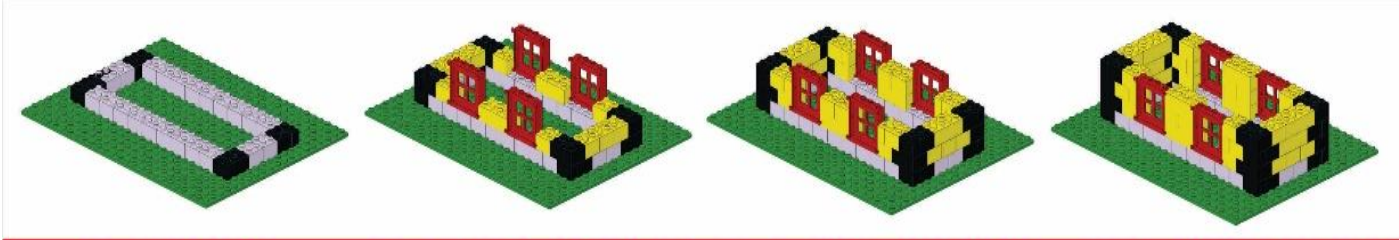

\section{1 - FUNDAÇÃO $\quad 02$ - 1a. FIADA - ALVENARIA 03 - 2a. FIADA - ALVENARIA 04 -3a. FIADA - ALVENARIA}

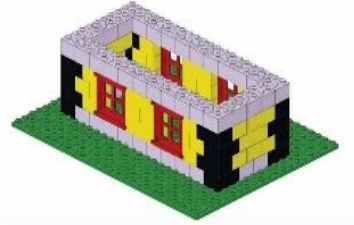

05 - CINTA SUPERIOR

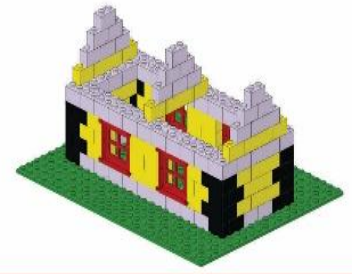

06 - OITÃO

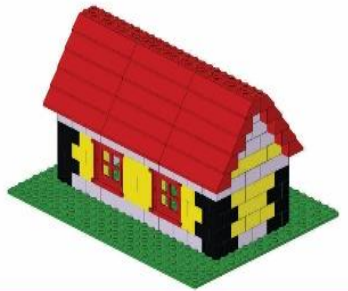

07 - COBERTA - CASA COMPLETA

Fonte: Autores, 2019.

\subsubsection{Linha de balanço}

Para programação da obra com linha de balanço, fez-se necessário o cálculo das durações de cada serviço com o levantamento do tempo médio da montagem de cada peça descritos na Tabela 1.

Tabela 1: Tempo de cada peça.

\begin{tabular}{lcc}
\hline Código & Tipo & Tempo estimado (s) \\
\hline $1 \times 2 \mathrm{P}$ & 1,33 \\
$1 \times 4 \mathrm{P}$ & 1,32 \\
2x2P & \\
*Janela & \\
*Telha & \\
\end{tabular}

Fonte: Autores, 2019. 
Research, Society and Development, v. 9, n. 7, e525974425, 2020

(CC BY 4.0) | ISSN 2525-3409 | DOI: http://dx.doi.org/10.33448/rsd-v9i7.4425

Objetivando-se mensurar o tempo médio de manuseio e utilização das peças (tempo estimado), foi realizada a montagem de blocos de 5, 10, 20 e 30 peças com a mesma tipologia cada. O resultado está apresentado na Figura 3. Os tempos consumidos pela janela e pela telha corresponderam a um percentual do tempo consumido para a execução da $1^{\mathrm{a}}$ fiada da alvenaria e da coberta, respectivamente.

Figura 3: Tempo médio de cada unidade tipológica.

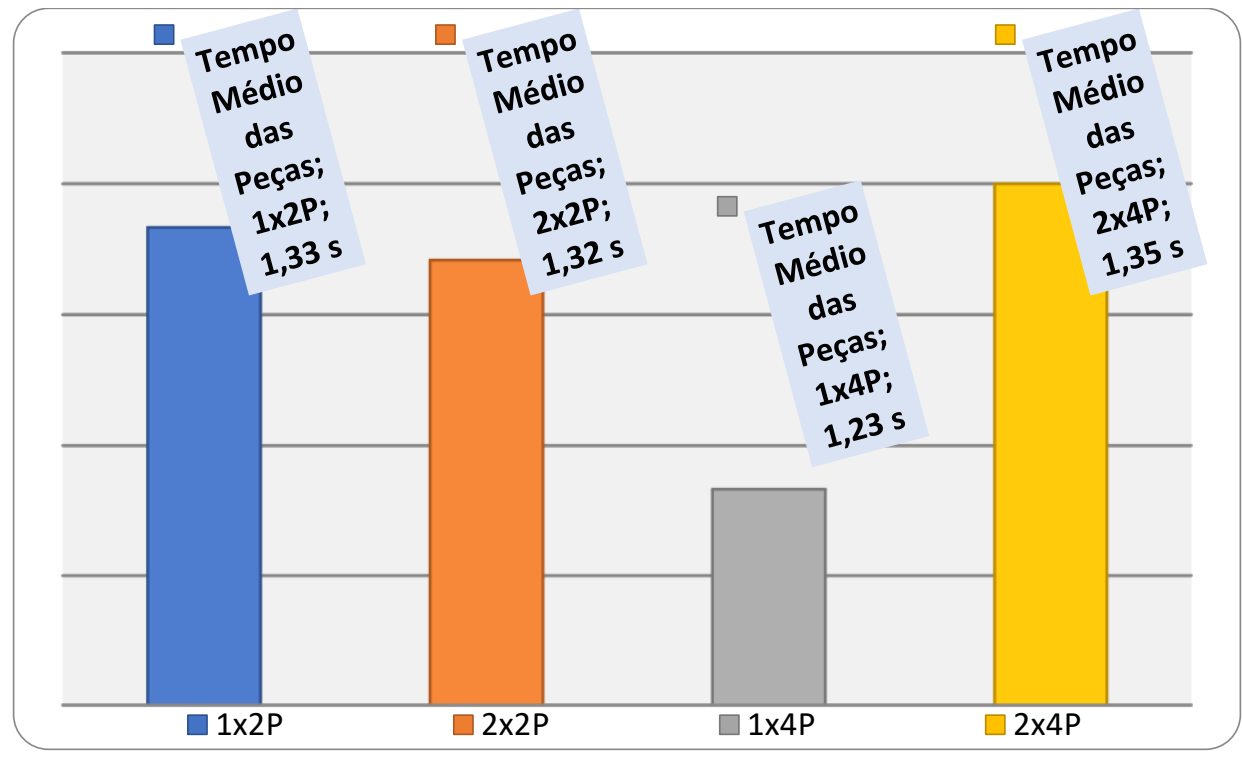

Fonte: Autores, 2019.

Com a quantificação do número de peças de cada serviço e obtenção prévia do tempo estimado de montagem de cada peça calculado pelo tempo médio de montagem de uma dupla de alunos, definiu-se o tempo estimado total de cada serviço e produtividade estimada, descritos na Tabela 2. 
Research, Society and Development, v. 9, n. 7, e525974425, 2020

(CC BY 4.0) | ISSN 2525-3409 | DOI: http://dx.doi.org/10.33448/rsd-v9i7.4425

Tabela 2: Tempo estimado de cada serviço.

\begin{tabular}{|c|c|c|c|c|c|c|}
\hline Item & Serviço & Alunos & Tipo & Peças & $\begin{array}{c}\text { Tempo } \\
\text { Estimado } \\
\text { Unitário }\end{array}$ & $\begin{array}{c}\text { Produtividade } \\
\text { Estimada }\end{array}$ \\
\hline \multirow{2}{*}{1} & \multirow{2}{*}{ Fundação } & \multirow{2}{*}{1} & $2 \times 2 P$ & 22 & $27,1 \mathrm{~s}$ & \multirow{2}{*}{-} \\
\hline & & & $1 \times 2 P$ & 12 & $16,0 \mathrm{~s}$ & \\
\hline \multicolumn{5}{|c|}{ SubTotal 1} & $43,1 \mathrm{~s}$ & $43 \mathrm{~s} / \mathrm{HH}$ \\
\hline \multirow{3}{*}{2} & \multirow{3}{*}{$1^{\mathrm{a}}$ Fiada } & \multirow{3}{*}{2} & Janela* & 4 & & \multirow{3}{*}{-} \\
\hline & & & $1 \mathrm{x} 4 \mathrm{P}$ & 8 & $10,6 \mathrm{~s}$ & \\
\hline & & & $1 \times 2 P$ & 24 & $32,0 \mathrm{~s}$ & \\
\hline \multicolumn{5}{|c|}{ SubTotal 2} & $46,0 \mathrm{~s}$ & $23 \mathrm{~s} / \mathrm{HH}$ \\
\hline \multirow{2}{*}{3} & \multirow{2}{*}{$2^{\mathrm{a}}$ Fiada } & \multirow{2}{*}{2} & $1 \times 4 P$ & 4 & $5,3 \mathrm{~s}$ & \multirow{2}{*}{-} \\
\hline & & & $1 \times 2 P$ & 32 & $42,7 \mathrm{~s}$ & \\
\hline \multicolumn{5}{|c|}{ SubTotal 3} & $48,0 \mathrm{~s}$ & $24 \mathrm{~s} / \mathrm{HH}$ \\
\hline \multirow{2}{*}{4} & \multirow{2}{*}{$3^{\mathrm{a}}$ Fiada } & \multirow{2}{*}{2} & $1 \times 4 P$ & 8 & $10,6 \mathrm{~s}$ & \multirow[b]{2}{*}{ - } \\
\hline & & & $1 \times 2 P$ & 24 & $32,0 \mathrm{~s}$ & \\
\hline \multicolumn{5}{|c|}{ SubTotal 4} & $42,6 \mathrm{~s}$ & $21 \mathrm{~s} / \mathrm{HH}$ \\
\hline \multirow{2}{*}{5} & \multirow{2}{*}{ Cinta } & \multirow{2}{*}{1} & $2 \times 2 P$ & 18 & $22,2 \mathrm{~s}$ & \multirow[b]{2}{*}{-} \\
\hline & & & $1 \mathrm{X} 2 \mathrm{P}$ & 20 & $26,7 \mathrm{~s}$ & \\
\hline \multicolumn{5}{|c|}{ SubTotal 5} & $48,9 \mathrm{~s}$ & $49 \mathrm{~s} / \mathrm{HH}$ \\
\hline \multirow{3}{*}{6} & \multirow{3}{*}{ Coberta } & \multirow{3}{*}{1} & Telha* & 30 & & \multirow{3}{*}{-} \\
\hline & & & $1 \mathrm{X} 4 \mathrm{P}$ & 4 & $5,3 \mathrm{~s}$ & \\
\hline & & & $1 \times 2 P$ & 22 & $29,3 \mathrm{~s}$ & \\
\hline \multicolumn{5}{|c|}{ SubTotal 6} & $42,4 \mathrm{~s}$ & $42 \mathrm{~s} / \mathrm{HH}$ \\
\hline \multicolumn{5}{|c|}{ TOTAL } & $271,0 \mathrm{~s}$ & - \\
\hline
\end{tabular}

Fonte: Autores, 2019.

Foi definido do ritmo das atividades de modo a entregar uma casa pronta por vez e possibilitar a puxada das atividades. Com estas definições elaborou-se a linha de balanço da obra (Figura 4). 
Research, Society and Development, v. 9, n. 7, e525974425, 2020

(CC BY 4.0) | ISSN 2525-3409 | DOI: http://dx.doi.org/10.33448/rsd-v9i7.4425

Figura 4: Linha de balanço estimada.

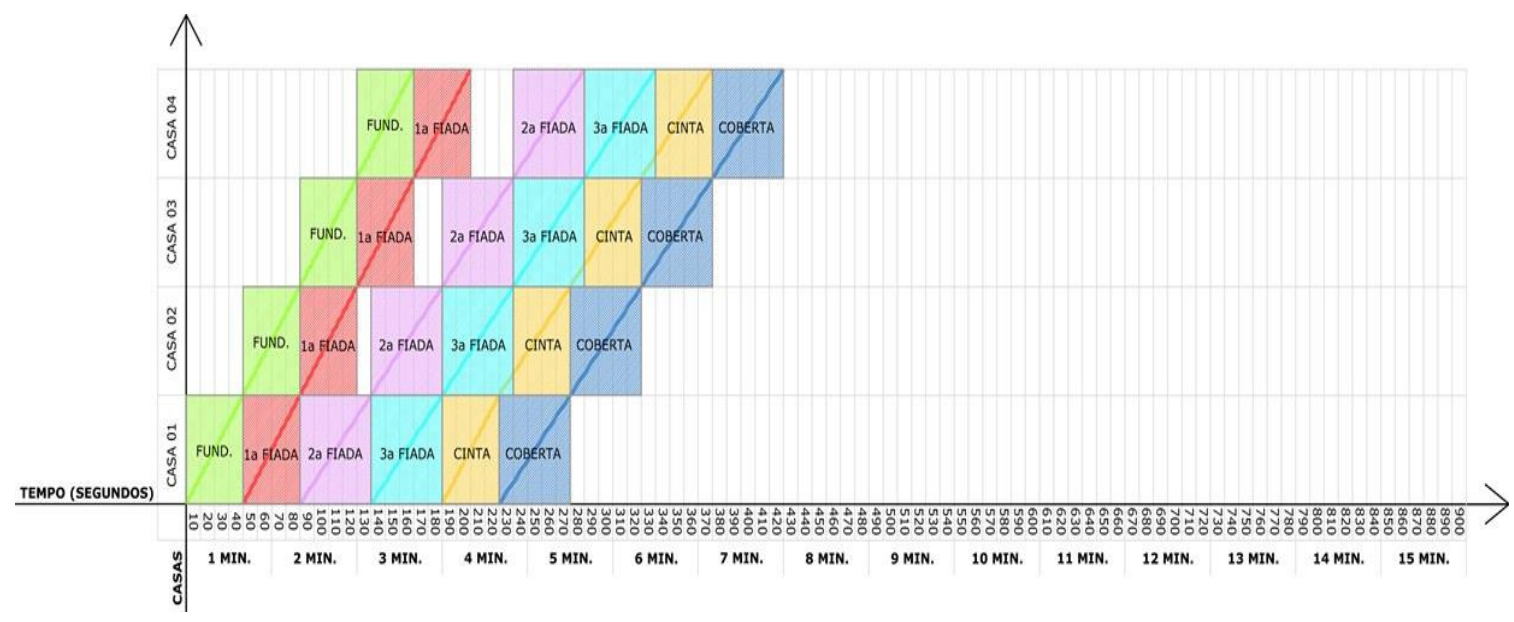

Fonte: Autores, 2019.

\subsubsection{Suprimento}

O estoque de materiais (Figura 5) guardando a distância de três metros do canteiro de obra, foi organizado em pallets divididos para a execução de cada casa da seguinte maneira: fundação; alvenaria $1^{\text {a }}$. fiada e esquadria; alvenaria $2^{\mathrm{a}}$ Fiada; alvenaria $3^{\mathrm{a}}$ Fiada; cinta de amarração e coberta. A Figura 5 apresenta o estoque dos pallets no setor de suprimentos e na Figura 6 está representado um pallet de alvenaria $2^{\mathrm{a}}$ fiada.

Figura 5: Suprimento em pallets.

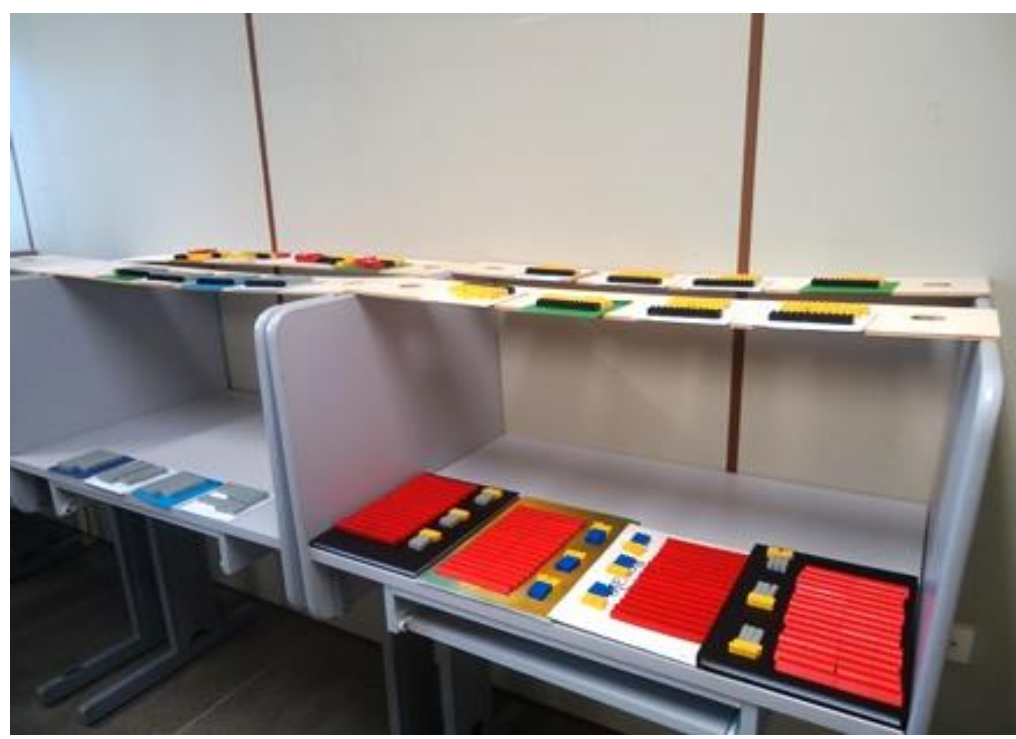

Fonte: Autores, 2019. 
Figura 6: Pallet alvenaria $2^{\mathrm{a}}$ fiada.

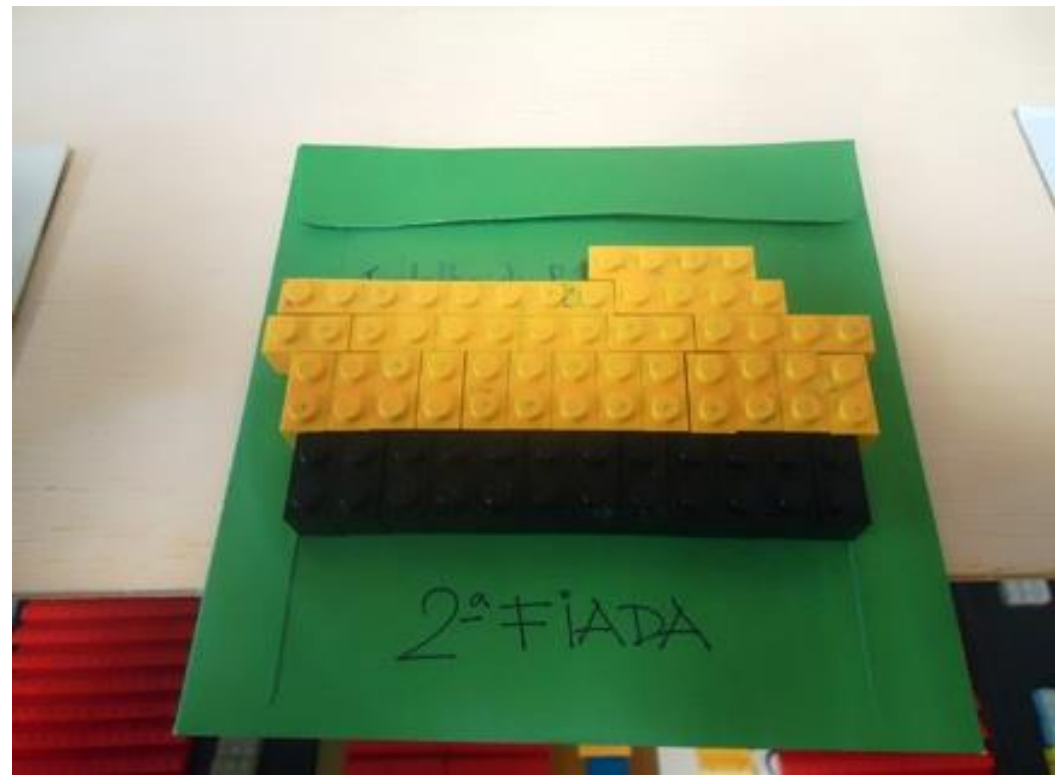

Fonte: Autores, 2019.

A solicitação e controle do fornecimento de material para as atividades foi organizado através de kambans de transporte e material (Figura 7) e o chão de fábrica foi definido guardando a distância de um metro entre cada casa a ser construída para simular situação real de canteiro de obra e permitir espaço para trabalho das equipes de montagem (Figura 8).

Figura 7: Atividades organizado em Kambans.

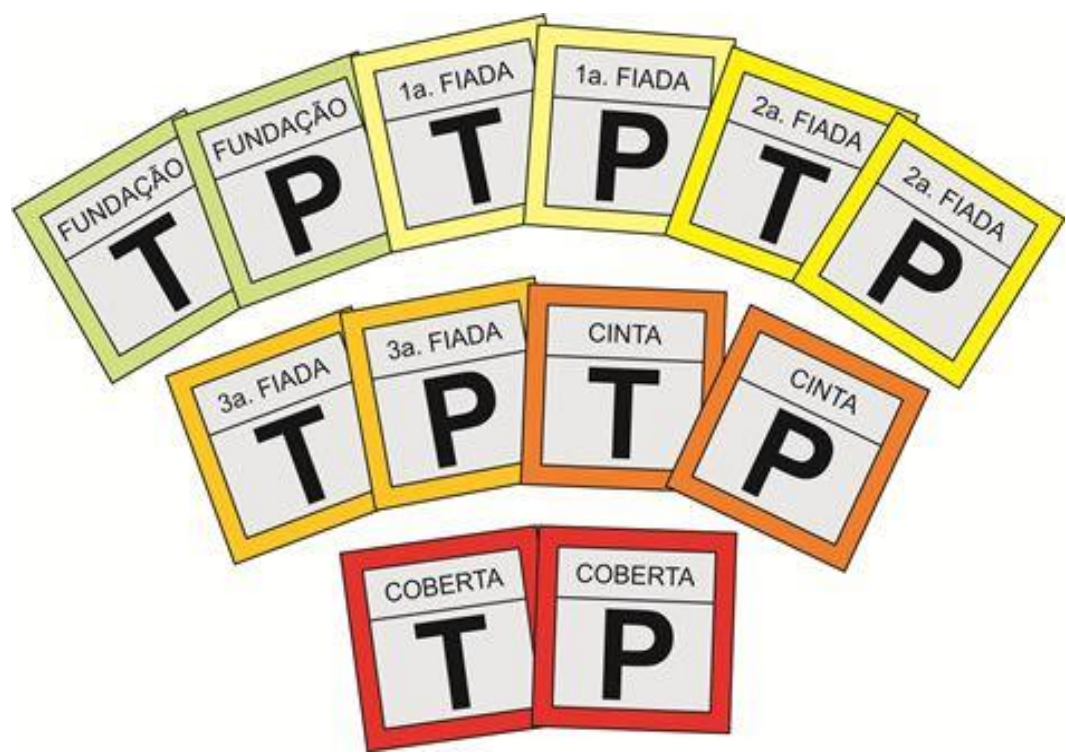

Fonte: Autores, 2019. 
Figura 8: Equipe na montagem.

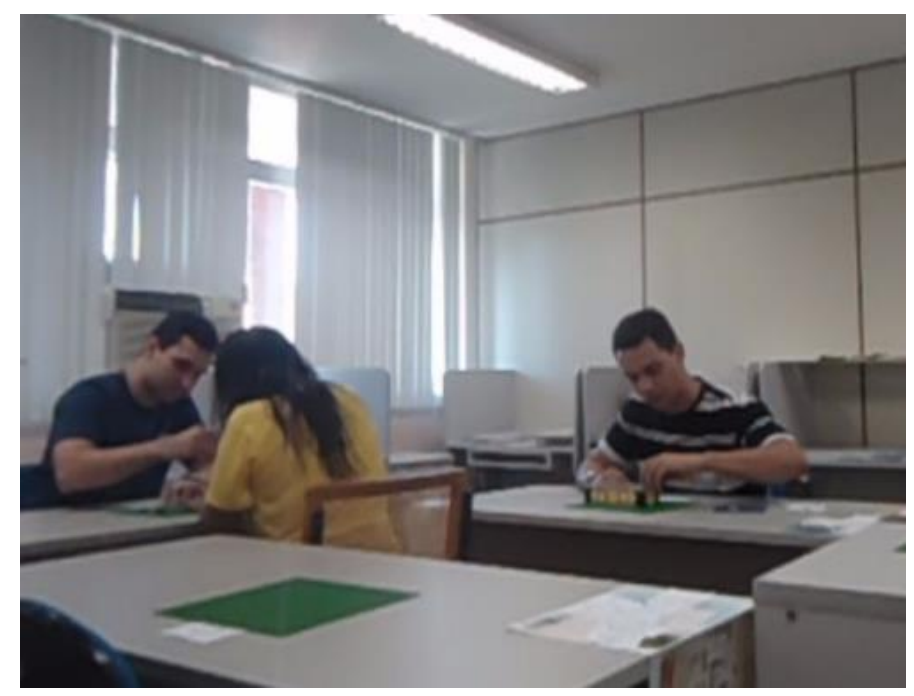

Fonte: Autores, 2019.

Na Figura 9 o canteiro de obra com as quatro casas montadas e na Figura 10 detalhe de uma casa pronta.

Figura 9: Canteiro 4 casas.

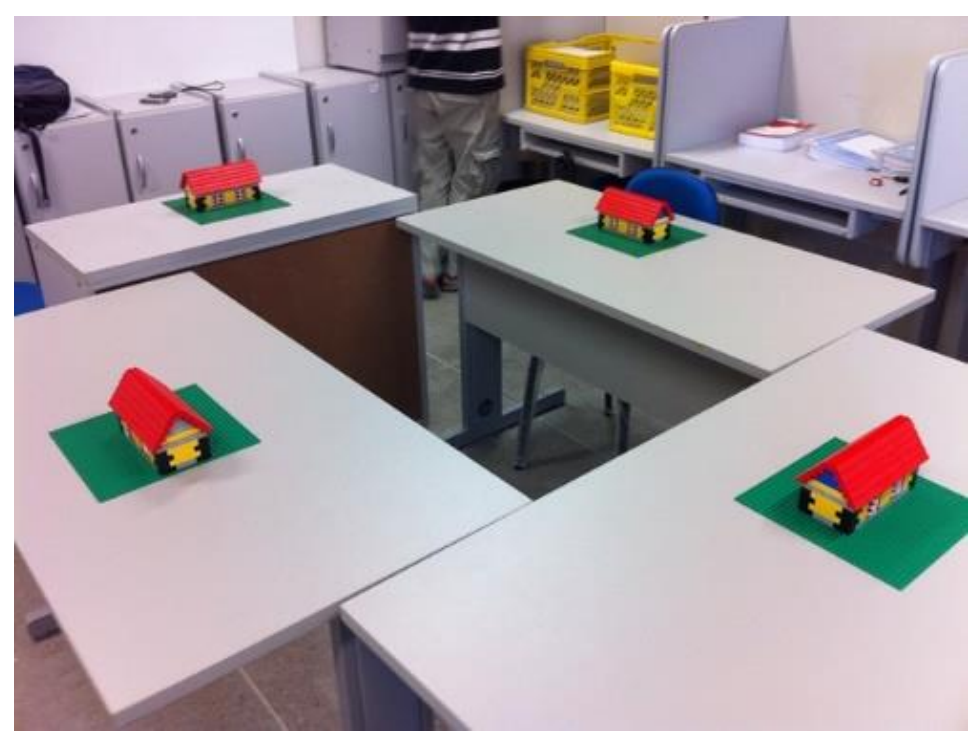

Fonte: Autores, 2019. 
Figura 10: Casa pronta.

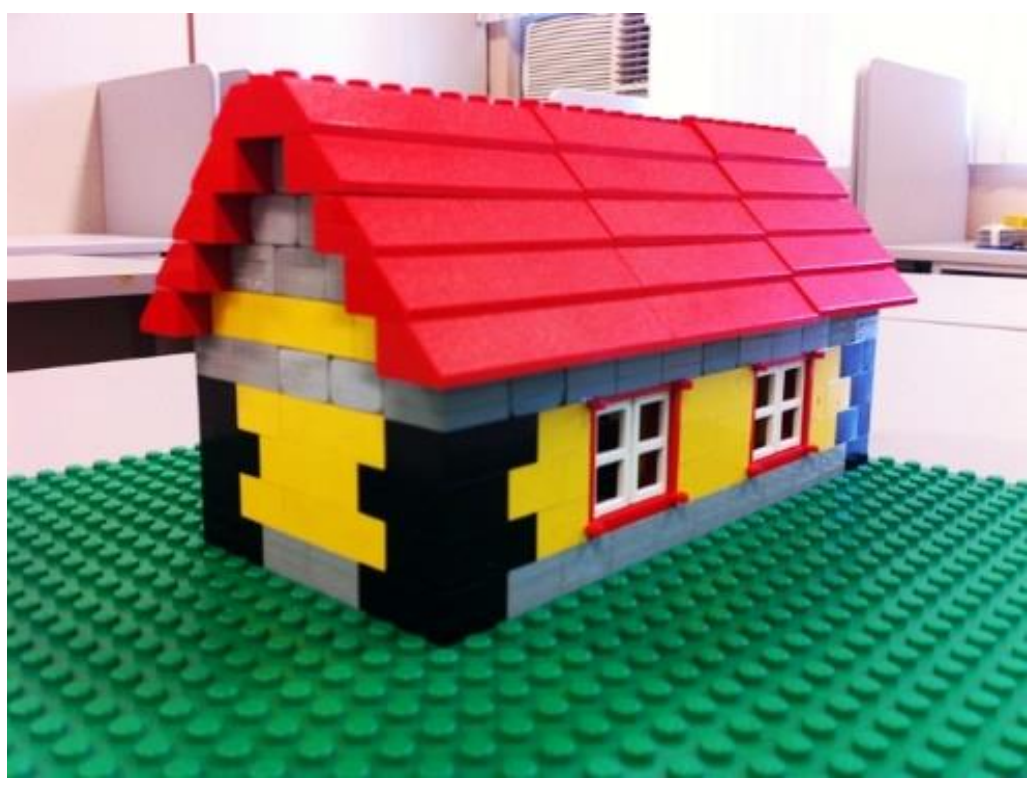

Fonte: Autores, 2019.

\section{Resultados e Discussão}

\subsection{Produtividade real e linha de balanço com tempo real de execução}

Percebe-se pela Tabela 3 que os serviços de fundação, cinta e coberta foram os principais responsáveis pela divergência entre o tempo de execução estimado e o tempo real, sendo suas produtividades estimadas, respectivamente, em aproximadamente $58 \%$, $48 \%$ e $24 \%$ dos valores reais. Nota-se também que o tempo médio de transporte dos pallets do setor de suprimentos até o canteiro representa $12 \%$ do tempo total real, fato este que foi desconsiderado durante o processo de estimativa. 
Research, Society and Development, v. 9, n. 7, e525974425, 2020

(CC BY 4.0) | ISSN 2525-3409 | DOI: http://dx.doi.org/10.33448/rsd-v9i7.4425

Tabela 3: Produtividade estimada x real.

\begin{tabular}{|c|c|c|c|c|c|c|c|c|c|c|c|c|c|}
\hline \multirow{2}{*}{ Item } & \multirow{2}{*}{ Serviço } & \multirow{2}{*}{ Qre de lquipes } & \multirow{2}{*}{ Tipo } & \multirow{2}{*}{ Qde } & \multirow{2}{*}{$\begin{array}{c}\text { Tempo Etinado } \\
\text { Unitifio }\end{array}$} & \multirow{2}{*}{$\begin{array}{c}\text { Produtvidade } \\
\text { Gst-ads } \\
\end{array}$} & \multicolumn{6}{|c|}{ Temp Real Unitáio (Medio) } & \multirow{2}{*}{\begin{tabular}{|c} 
Produtividade \\
Real
\end{tabular}} \\
\hline & & & & & & & Decuchito & $\%$ & RANSFORTE & $\%$ & TOTAL & $\%$ & \\
\hline \multirow{2}{*}{1} & \multirow{2}{*}{ RUNDAC,AO } & \multirow{2}{*}{1} & $2 \times 29$ & 22 & $27,1 \mathrm{~s}$ & \multirow{2}{*}{ - } & \multirow[b]{2}{*}{ 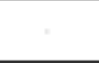 } & \multirow{2}{*}{$85 \%$} & \multirow[b]{2}{*}{ t } & \multirow{2}{*}{$15 \%$} & \multirow{2}{*}{ - } & \multirow{2}{*}{$15 \%$} & \multirow{2}{*}{ - } \\
\hline & & & $1 \times 29$ & 12 & $16,0 \mathrm{~s}$ & & & & & & & & \\
\hline \multicolumn{5}{|c|}{ SubTotal1 } & 43.1s & $435 / H A$ & 74753 & $*$ & 12,753 & + & 8,58 & + & $\mathrm{TS/HH}$ \\
\hline \multirow{3}{*}{2} & \multirow{3}{*}{ PRADA } & \multirow{3}{*}{2} & JANQA: & \begin{tabular}{l|l}
4 \\
\end{tabular} & & \multirow{3}{*}{$\cdot$} & \multirow{3}{*}{$=$} & \multirow{3}{*}{85} & \multirow{3}{*}{. } & \multirow{3}{*}{$15 \%$} & \multirow{3}{*}{ - } & \multirow{3}{*}{$12 \%$} & \\
\hline & & & $1 \times 42$ & 8 & $10,6 \mathrm{~s}$ & & & & & & & & - \\
\hline & & & $1 \times 29$ & 24 & $32,0 \mathrm{~s}$ & & & & & & & & \\
\hline & & & & bTotal2 & $46,0 \mathrm{~s}$ & $\mathrm{Z}_{\mathrm{S} / \mathrm{HH}}$ & 61,75 & + & 10,753 & 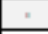 & $n, 0 \mathrm{~s}$ & + & $31 \mathrm{~s} / \mathrm{HH}$ \\
\hline 3 & 20 pana & 2 & $1 \times 49$ & \begin{tabular}{|l|}
4 \\
\end{tabular} & $5,3 \mathrm{~s}$ & 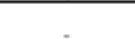 & . & 898 & 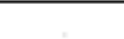 & $118 \mathrm{~s}$ & . & 108 & . \\
\hline 3 & ERAAUA & 2 & $1 \times 29$ & 32 & $42,7 \mathrm{~s}$ & - & 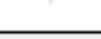 & 2093 & 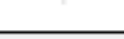 & 117 & ${ }^{-}$ & $12 \pi$ & - \\
\hline & & & & bTotal3 & $480 \mathrm{~s}$ & $24 s / H H$ & $62,5 \mathrm{~s}$ & 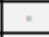 & $8,0 \mathrm{~s}$ & 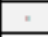 & 20.58 & + & $31 \mathrm{~s} / \mathrm{HH}$ \\
\hline 4 & Y pana & 2 & $1 \times 49$ & 8 & $10.6 \mathrm{~s}$ & 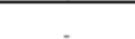 & . & $838 \mathrm{~s}$ & 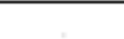 & 185 & . & nos. & . \\
\hline 4 & STHAUA & 2 & $1 \times 29$ & 24 & $32,0 \mathrm{~s}$ & - & te & & 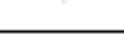 & & - & Wh & - \\
\hline & & & & bTotal4 & 42,68 & $21 s / 4 H$ & $49,5 s$ & 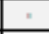 & $10,5 s$ & $=$ & $600 \mathrm{~s}$ & $\cdot$ & $\mathrm{S}_{\mathrm{s} / \mathrm{HH}}$ \\
\hline 5 & CNTS & 1 & $2 \times 29$ & 18 & $22,2 \mathrm{~s}$ & 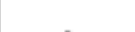 & & $=08$ & & 136 & & pog & 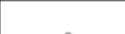 \\
\hline 3 & CNIA & 1 & $1 \times 2 \%$ & 20 & $26,7 \mathrm{~s}$ & - & te & $\infty$ & te & 10 & - & $16 \pi$ & - \\
\hline & & & & bTotals & 4895 & $49 \mathrm{~s} / \mathrm{HH}$ & 101,55 & $\cdot$ & $13,75 \mathrm{~s}$ & $=$ & 115,258 & $\cdot$ & $102 s / 414$ \\
\hline & & & TQHA & 30 & & & & & & & & & \\
\hline 6 & COACRTA & 1 & $1 \times 49$ & 4 & $5,3 \mathrm{~s}$ & - & & 925 & & 85 & - & $32 \%$ & - \\
\hline & & & $1 \times 29$ & 22 & $29,3 \mathrm{~s}$ & & & & & & & & \\
\hline & & & & bTotal6 & $42,4 \mathrm{~s}$ & $425 / \mathrm{HH}$ & $177,75 \mathrm{~s}$ & $=$ & $15,25 \mathrm{~s}$ & $=$ & $193,0 \mathrm{~s}$ & $\cdot$ & $178, / H A$ \\
\hline & & & & TOTAL & 71,05 & $\cdot$ & $527,25 \mathrm{~s}$ & $88 \%$ & $71,0 \mathrm{~s}$ & 126 & 598255 & $100 \%$ & $\cdot$ \\
\hline
\end{tabular}

Fonte: Autores, 2019.

Na Figura 11 está representada a linha de balanço real onde foi ajustado aos tempos reais de execução das atividades.

Figura 11: Linha de balanço real.

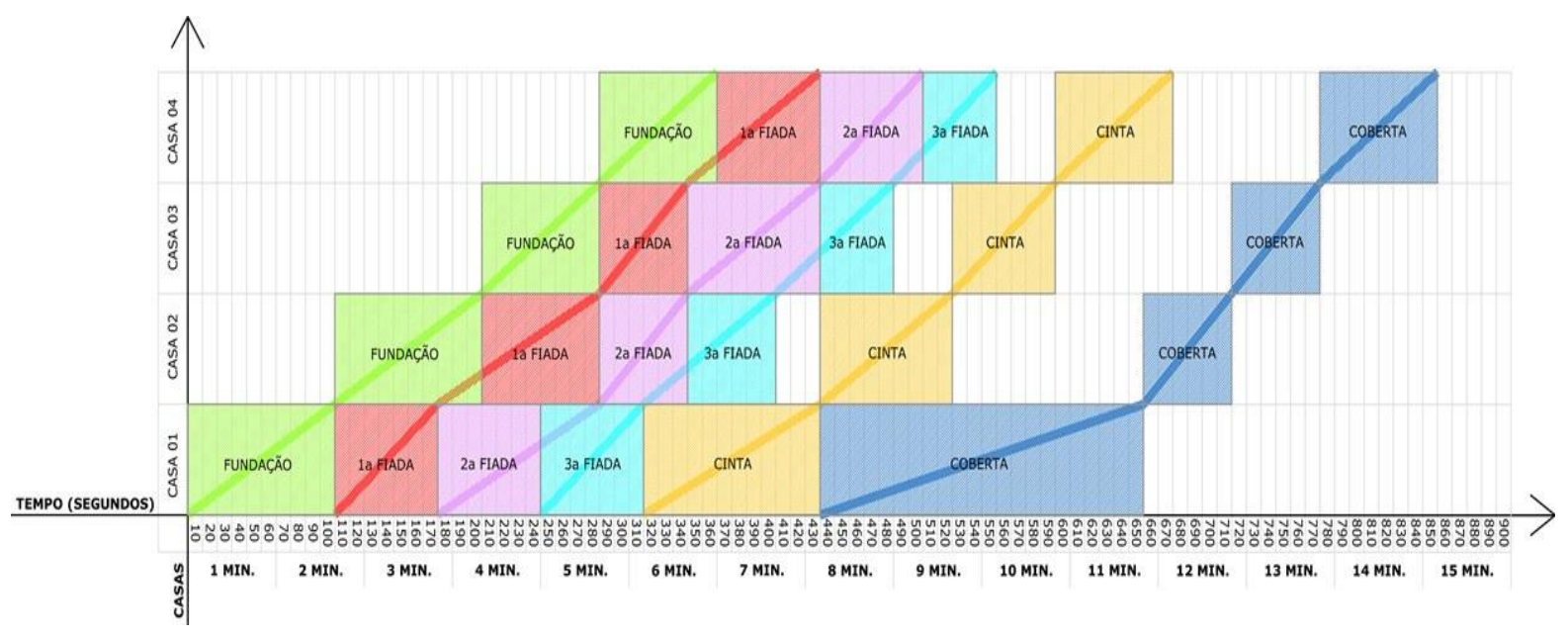

Fonte: Autores, 2019.

Os tempos de execução e transporte estão descritos na Figura 12, no qual representa fundação de transporte, fundação execução, fiada transporte e fiada execução. 
Figura 12: Gráfico transporte e execução.

04 Casas - Transporte e Execução

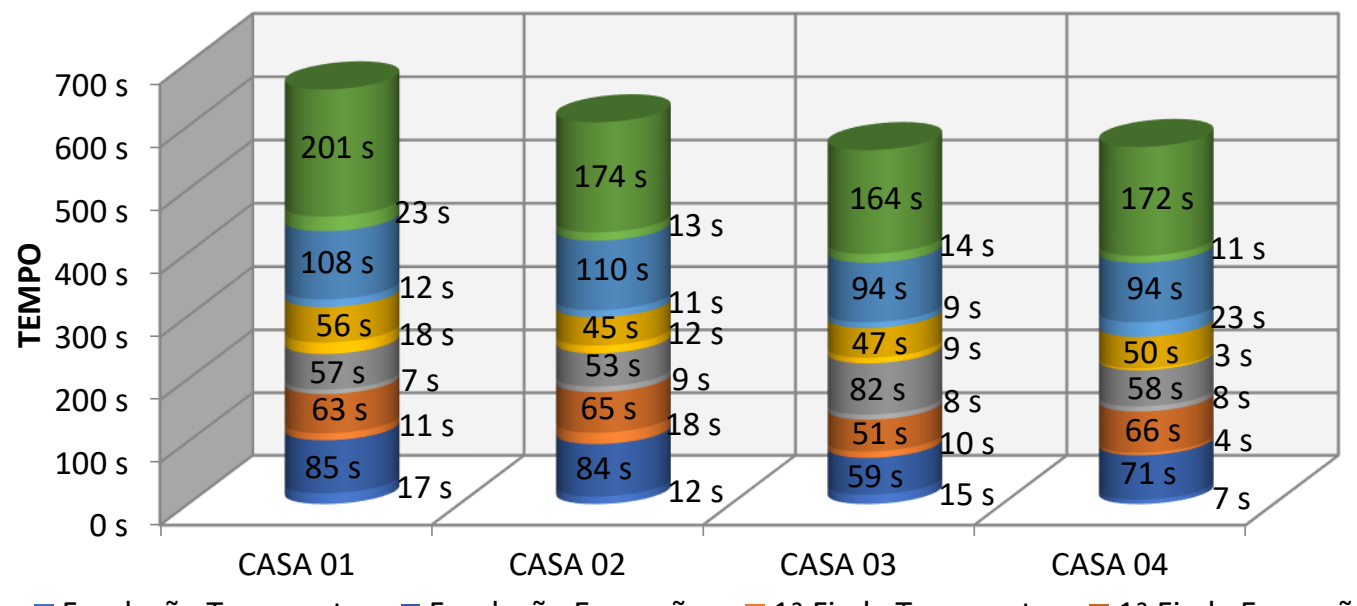

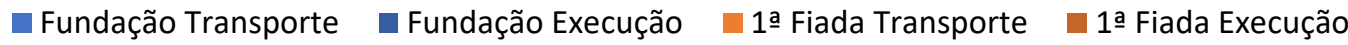

Fonte: Autores, 2019.

Os serviços de fundação estimados em 43 segundos variaram entre 59 e 85 segundos, já os serviços de $2^{\mathrm{a}}$. fiada de alvenaria estimados em 48 segundos variaram entre 53 e 82 segundos. Apesar das equipes terem feito um treinamento inicial de montagem das peças nas atividades, quando as casas entraram no ritmo de produção os atrasos ocorreram por retrabalhos e devido a própria dinâmica da produção em série.

\subsection{Linha de balanço estimada $X$ tempo real de execução}

Na Figura 13 temos um comparativo entre as linhas de balanço estimada e real. Observou-se aqui que todos os ritmos das atividades não ocorreram como previsto especialmente os serviços de coberta que tiveram a dificuldade de montar as empenas (oitão) e telhas e ocasionaram bastante atraso na conclusão da casa. 
Research, Society and Development, v. 9, n. 7, e525974425, 2020

(CC BY 4.0) | ISSN 2525-3409 | DOI: http://dx.doi.org/10.33448/rsd-v9i7.4425

Figura 13: Gráfico transporte e execução.

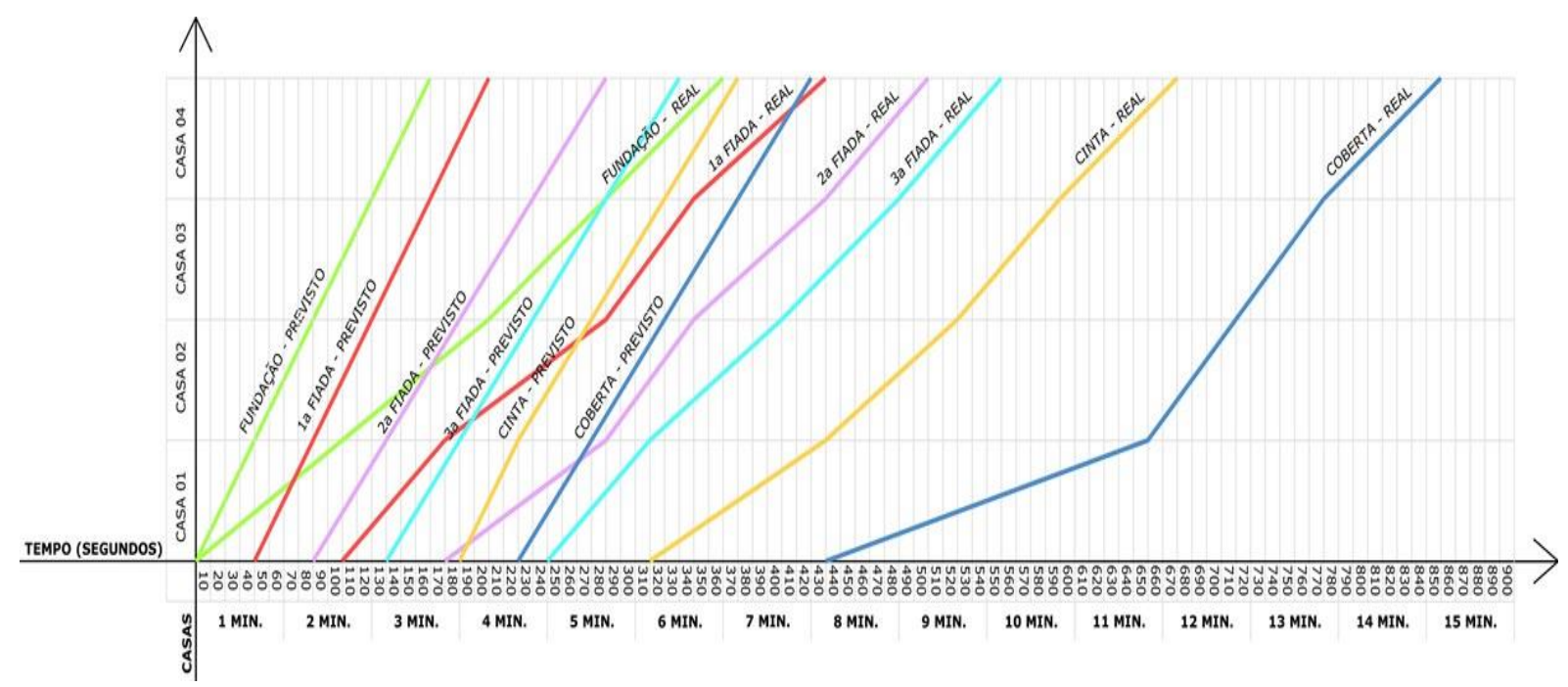

Fonte: Autores, 2019.

Na Figura 14 observamos os tempos de serviços previstos e realizados através do gráfico. O serviço de coberta teve a duração $100 \%$ maior que o tempo previsto inicialmente para execução.

Figura 14: Gráfico tempo das atividades.

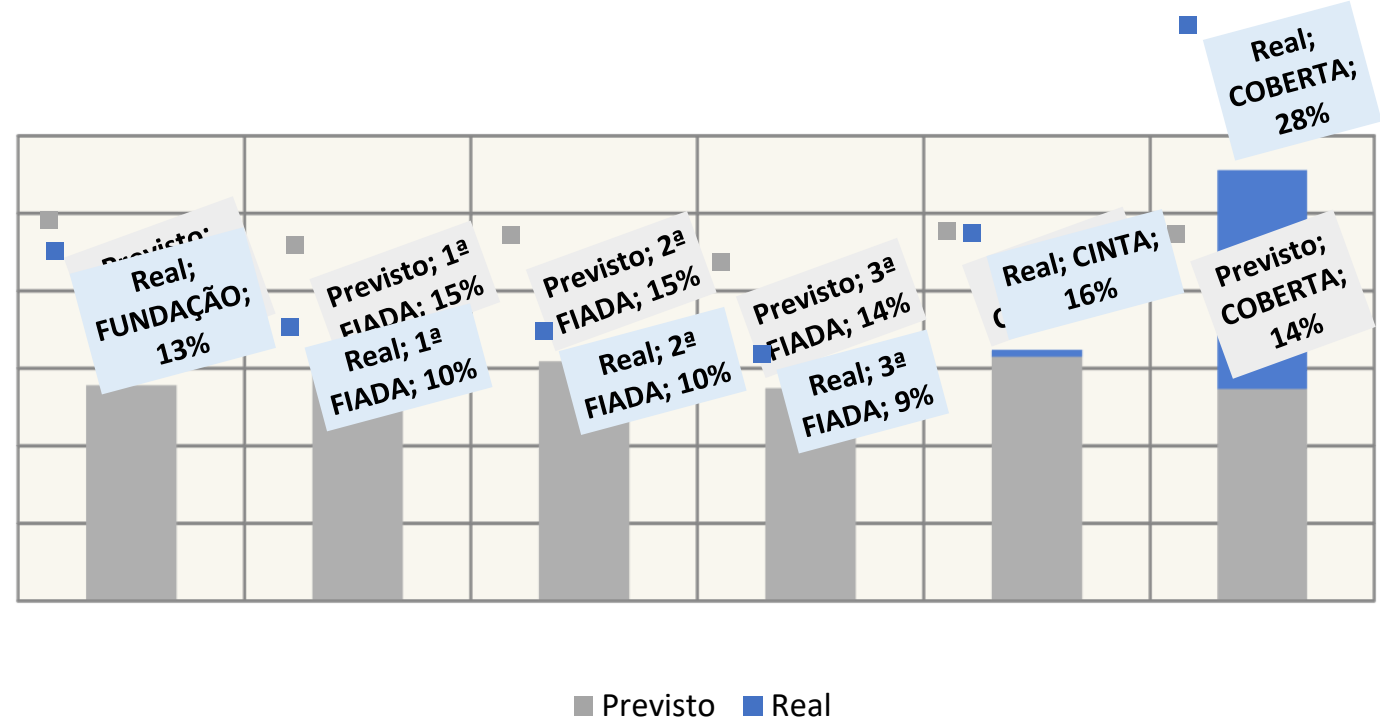

Fonte: Autores, 2019. 


\subsection{Efeito aprendizagem}

Encontra-se descrito na Figura 15 o efeito aprendizagem $\mathrm{x}$ fadiga das atividades. Observou-se que o efeito aprendizagem foi mais evidente nos serviços de fundação, $3^{\mathrm{a}}$ fiada e coberta e o de fadiga nos serviços de $2^{\mathrm{a}}$ fiada.

Figura 15: Gráfico aprendizagem x fadiga.

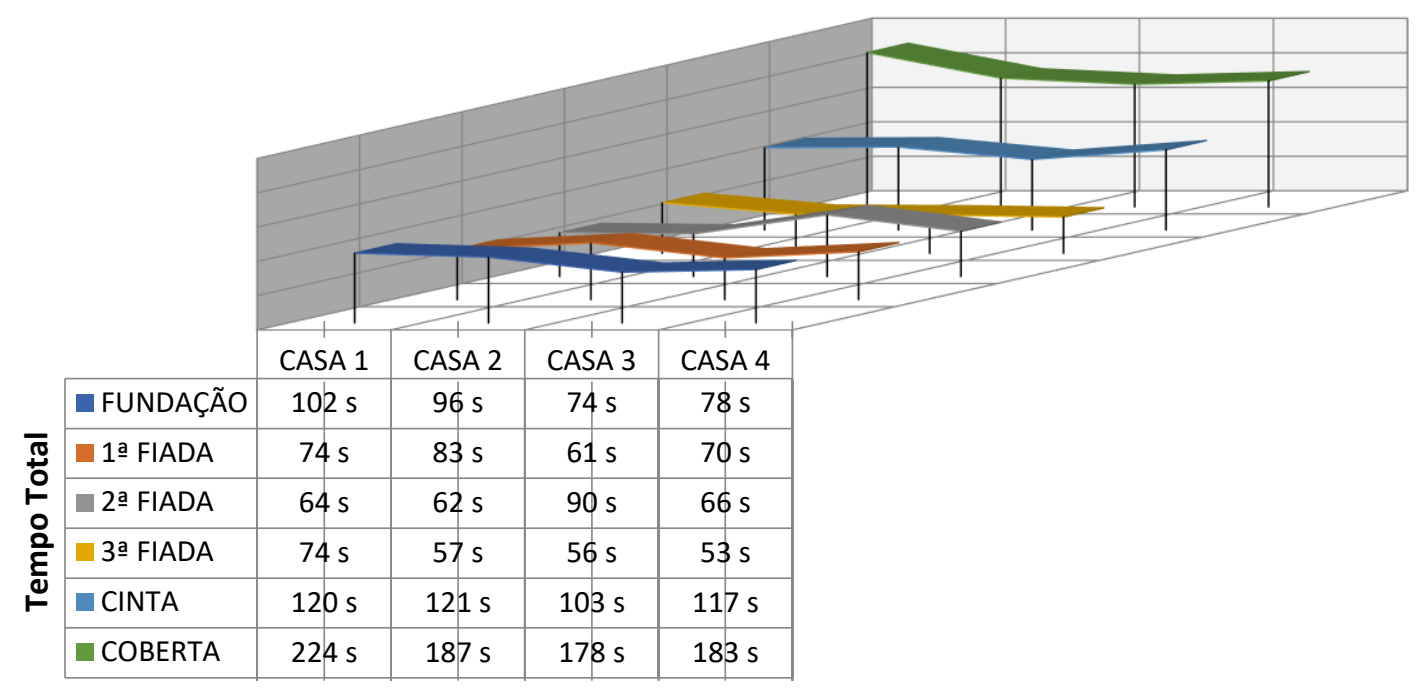

Fonte: Autores, 2019.

\section{Considerações Finais}

O presente trabalho apresentou o relato de uma experiência na qual se utilizou um jogo como complemento ao processo de aprendizado em sala de aula. A partir do jogo realizado, os alunos puderam compreender melhor as características da técnica da linha de balanço, bem como os procedimentos para realizar a programação de maneira adequada. Os conceitos de programação de obra, ritmo das atividades, fluxo contínuo foram assimilados pelos alunos neste processo de simulação. A partir dos resultados obtidos é possível observar um aumento no efeito aprendizagem e em contrapartida uma diminuição da produtividade pelo efeito fadiga, já que algumas atividades foram repetidas pelas equipes por diversas vezes.

Quando saímos do campo estimado para o campo real, percebemos que a alteração nos tempos é referente aos efeitos aqui citados, tempo de transporte, alguns retrabalhos, sendo extremamente necessária a medição dos tempos de transporte para o controle do tempo real das 
atividades. Na construção civil a inovação passa também por um melhor planejamento da obra, em todos os seus processos produtivos para gerar eficiência na execução e valor para o cliente.

\section{Referências}

Depexe, M. D., Dorneles, J. B., Kemmer, S. L., Silveira, J. P., Santos, D. G., \& Heineck, L. (2006). Aprendizado da técnica de programação da linha de balanço por meio de jogos didáticos. Encontro Nacional de Tecnologia no Ambiente Construído, 11, 2209-2218.

Doyle, D., \& Brown, F. W. (2000). Using a business simulation to teach applied skills-the benefits and the challenges of using student teams from multiple countries. Journal of European industrial training. p. 330-336.

Dressler, J. (1974). Stochastic Sheduling of Linear Construction Sites. Journal of the Construction Division. p. 571-587.

Lumsden, C. E. (1968). Nervous tissue in culture. In The structure and function of nervous tissue. p. 67-140.

Lutz, J. D. (1990). Planning of Linear Construction Using Simulation and Line of Balance (Doctoral dissertation, Ph. D. Dissertation, School of Civil Engineering, Purdue University, W. Lafayette, IN).

Mendes Jr, R. (2000). Jogo de programação da construção de edifícios na internet. In: XXVIII Congresso Brasileiro de Educação em Engenharia.p.1-9.

Rauch-Geelhaar, C., Jenke, K., \& Thurnes, C. M. (2003). Gaming in industrial managementquality and competence in advanced training. Production Planning \& Control, 14(2), 155-165.

Santos, D.G., Borges, V.P., Prado, R.L. \& Heineck, L. F. M. (2002). O ensino de linha de balanço e variabilidade através de um jogo didático. In: Encontro Nacional de Tecnologia do Ambiente Construído, p. 767-776. 
Sumichrast, R. T., \& Russell, R. S. (1990). Evaluating mixed-model assembly line sequencing heuristics for just-in-time production systems. Journal of Operations Management, 9(3), 371390.

Vargas, C. L. S. A., Krüger, J. A., Heineck, L. F. M., \& Coelho, R. D. Q. (1998). Avaliação de produtividade e de perdas na construção civil: simulação utilizando modelo reduzido para demonstrar as vantagens do uso da linha de balanço na programação da obra e de inovações tecnológicas no canteiro. In: Encontro Nacional de Tecnologia do Ambiente Construído, 159168.

Vargas, C. L. S. A., Mendes Júnior, R., Heineck, L. F. M., \& Krüger, J. A. (1998). Programação e controle de atividades repetitivas na execução de obras com modelo reduzido utilizando a técnica da linha de balanço. In: Encontro Nacional de Engenharia de Produção, p. 21-28.

\title{
Porcentagem de contribuição de cada autor no manuscrito
}

\author{
Bruno Barbosa de Oliveira - 30\% \\ Luiz Fernando Mählmann Heineck - 20\% \\ Miguel Adriano Gonçalves Cirino - 10\% \\ João Marcos Pereira de Morais - 10\% \\ Eduarda Morais da Silva - 10\% \\ Eliezio Nascimento Barboza - 10\%
}

Synardo Leonardo de Oliveira Pereira - 10\% 